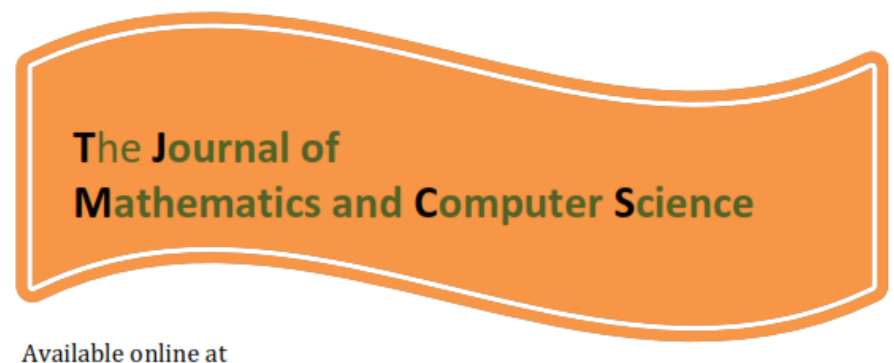

http://www.TJMCS.com

The Journal of Mathematics and Computer Science Vol. 4 No.3 (2012) 331 - 343

\title{
Taylor series method for the system of linear Volterra integro-differential equations
}

\author{
J. Rashidinia ${ }^{1}$, A. Tahmasebi \\ School of Mathematics, Iran University of Science and Technology \\ P. O. Box, 16846-13114, Tehran, Iran.
}

Received: February 2012, Revised: May 2012

Online Publication: July 2012

\begin{abstract}
A method to determine the numerical solution of system of linear Volterra integro-differential equations (IDEs) is proposed. The method obtains Taylor expansion for the exact solution of system of linear Volterra IDEs at initial point $x=0$. In addition, we introduce a procedure to obtain an approximation for Taylor expansion of the exact solution at $x \neq 0$. Moreover, error estimation of the proposed methods is presented. The efficiency and applicability of the presented methods is illustrated by some numerical examples.
\end{abstract}

Keywords: system of linear Volterra integro-differential equations; numerical solution; Taylor expansion; power series method; integral equation.

2000 AMS Subject Classification: 45J10; 65R99; $41 A 58$

\section{Introduction}

Integro-differential equations have been found to describe various kinds of phenomena, such as glassforming process, nano-hydrodynamics, drop wise condensation, and wind ripple in the desert [1-4]. There are several numerical and analytical methods for solving IDEs. Some different methods to solve integral and integro-differential equations are presented in [5]. [6] has used rationalized Haar functions method to solve the linear IDEs system. Linear IDEs system has been solved in [7] by using Galerkin methods with the hybrid Legendre and Block-Pluse functions on interval [0,1). In [8] an application of He's homotopy perturbation (HPM) method applied to solve of IDEs system. He's variational iteration method used for solving two systems of Volterra integro-differential equations [9]. Arikoglu et al. differential transform method (DTM) applied to both integro-differential and integral equation systems [10]. Biazar [11] proposed He's homotopy perturbation (HPM) method for system of integro-differential equations. A numerical method based on interpolation of unknown functions at distinct interpolation points has been introduced in [12] for solving linear IDEs system with initial values. Recently Biazar introduced a new modification of homotopy perturbation method (NHPM) to obtain the solution of linear IDEs system in [13]. Taylor expansion method has been used for solving IDE's in $[14,15]$. In this work, we developed and modified Taylor series method (TSM) introduced in [16], to solve system of linear Volterra IDEs in the following form

$$
E_{m}(x)+V_{m}(x)=f_{m}(x), \quad m=1(1) n, \quad x, t \in \Gamma=[0, b],
$$

where

$$
\begin{aligned}
& E_{m}(x)=\sum_{i=1}^{n} \sum_{j=0}^{\alpha_{m i}} p_{m i j}(x) y_{i}^{(j)}(x) \\
& V_{m}(x)=\sum_{i=1}^{n} \int_{0}^{x}\left(k_{m i}(x, t) \sum_{j=0}^{\beta_{m i}} y_{i}^{(j)}(t)\right) d t
\end{aligned}
$$

${ }^{1}$ Corresponding author. Tel./Fax :+982173220000.

E-mail addresses: rashidinia@iust.ac.ir, tahmasbi@iust.ac.ir. 
we consider the supplementary conditions

$$
\sum_{m=1}^{n} \sum_{j=0}^{\alpha_{j}-1} B_{m j r} y_{m}^{(j)}(0)=c_{r},
$$

for $r=1,2, \ldots, \beta$, where

$$
\alpha_{j}=\max _{1 \leq m \leq n}\left\{\alpha_{m j}\right\}, \quad \beta=\sum_{j=1}^{n} \alpha_{j} .
$$

Suppose that, the functions $f_{m}(x), k_{m i}(x, t)$ and $p_{m i j}(x)$ are analytic functions with respect to all arguments on the interval $[0, b]$. The purpose of this study is to find the solution of the system (1) in the following form

$$
\mathbf{y}_{i}(x)=\sum_{k=0}^{N} \frac{1}{k !} y_{i}^{(k)}(c)(x-c)^{k}, \quad i=1(1) n, \quad 0 \leq c \leq x
$$

where $y_{i}^{(k)}(c), k=0(1) N$, are Taylor coefficients to be determined.

\section{Taylor-series method}

For solving (1) with the initial conditions (2) by TSM, suppose that the solution of problem be in the following form

$$
\mathbf{y}_{k}(x)=e_{k 0}+e_{k 1} x+\ldots+e_{k \alpha_{k}} x^{\alpha_{k}}, \quad k=1(1) n,
$$

where, for $k=1(1) n, e_{k i}=\frac{y_{k}^{(i)}(0)}{i !}, i=0,1, \ldots, \alpha_{k}-1$ are known and $e_{k \alpha_{k}}$, is unknown to be determined. By substituting (3) into (1), we obtain the following system of algebraic equations

$$
\left(L_{i}^{(1)}(e)-b_{i}^{(1)}\right) x^{p_{i}}+Q_{i}\left(x^{p_{i}+1}\right)=0, \quad i=1(1) n,
$$

where $L_{i}^{(1)}(e)$, is linear combination of $e_{k \alpha_{k}}, k=1(1) n$, and $Q_{i}\left(x^{p_{i}+1}\right)$ is a polynomial of degree greater than non-negative integer $p_{i}$, for $i=1(1) n$. By neglecting $Q_{i}\left(x^{p_{i}+1}\right)$ in (4), we have the following linear algebraic system

$$
A^{(1)} E_{1}=b^{(1)},
$$

$A_{n \times n}^{(1)}$ and $b_{n \times 1}^{(1)}$ are known and $E_{1}=\left[e_{1 \alpha_{1}}, e_{2 \alpha_{2}}, \cdots, e_{n \alpha_{n}}\right]^{t}$ is an unknown vector. By neglecting linear dependent rows and columns in the linear algebraic system (5) and solving, we obtain the unknown parameters $e_{i_{1} \alpha_{i_{1}}}, e_{i_{2} \alpha_{i_{2}}}, \cdots, e_{i_{k} \alpha_{i_{k}}}$, where $S=\left\{i_{1}, i_{2}, \ldots, i_{k}\right\}$ is a subset of $T=\{1,2, \cdots, n\}$.

In the next step, we suppose that the solution of (1)-(2) has the following form

$$
\mathbf{y}_{k}(x)=e_{k 0}+e_{k 1} x+\ldots+e_{k j} x^{j}, \quad k=1(1) n,
$$

where

$$
j= \begin{cases}\alpha_{k}+1, & \text { if } k \in S, \\ \alpha_{k}, & \text { if } k \in T-S,\end{cases}
$$

and $e_{k j}, k=1(1) n$, are unknown parameters. Substituting (6) into (1), we have the following linear algebraic system

$$
\left(L_{i}^{(2)}(e)-b_{i}^{(2)}\right) x^{r_{i}}+Q_{i}\left(x^{r_{i}+1}\right)=0, \quad \text { for } \quad i=1(1) n,
$$

where

$$
r_{i}= \begin{cases}p_{i}+1, & \text { if } \quad i \in S, \\ p_{i}, & \text { if } \quad i \in T-S,\end{cases}
$$


and $L_{i}^{(2)}(e), i=1(1) n$, are linear combination of $e_{k j}, k=1(1) n$. Again, by neglecting $Q_{i}\left(x^{r_{i}+1}\right)$, we have the following linear algebraic system

$$
A^{(2)} E_{2}=b^{(2)},
$$

$A_{n \times n}^{(2)}$ and $b_{n \times 1}^{(2)}$ are known and $E_{2}$ is a unknown vector of $e_{k j}, k=1(1) n$. Neglecting the linear dependent rows and columns and solving, the unknown parameters $e_{i_{1}^{\prime} \alpha_{i_{1}^{\prime}}}, e_{i_{2}^{\prime} \alpha_{i_{2}^{\prime}}}, \cdots, e_{i_{k}^{\prime} \alpha_{i_{k}^{\prime}}}$, in (6) can be obtained. By repeating the above procedure the following power series is derived

$$
\mathbf{y}_{k}(x)=e_{k 0}+e_{k 1} x+e_{k 2} x^{2}+\ldots, \quad k=1(1) n .
$$

In order to approximate the exact solution $y_{k}(x)$, for $k=1(1) n$, we use the first $\rho$ terms of (11). Now, in the following theorem, we will prove that the above procedure which is given in (11), is Taylor expansion of exact solution of (1)-(2) at $x=0$.

Theorem 1: We assume that the functions $f_{m}(x), k_{m i}(x, t)$ and $p_{m i j}(x)$ in (1) are analytic functions with respect to all theirs arguments at $x=0$, and also we suppose that there exist $i, j$ such that $p_{m i j}(0) \neq 0$ for $m=1(1) n$. Then TSM gives Taylor expansion of the exact solution (1)-(2) at $x=0$.

Proof : We have to Prove that, in the $\sigma$ th step of the TSM, we obtain

$$
e_{i j}=\frac{y^{(j)}(0)}{(j) !}, \quad i \in\{1,2, \ldots, k\}, k \leq n
$$

For proving, we use induction. For $\sigma=1$, soppose $s_{m}, m=1(1) n$, are the smallest nonnegative integer such that, the following equations are't vanish at $x=0$

$$
\sum_{i=1}^{n} \sum_{j=0}^{\alpha_{m i}} \sum_{k=0}^{s_{m}} \eta_{k} p_{m i j}^{\left(s_{m}-k\right)}(x) y_{i}^{(j+k)}(x)+\chi_{m}^{\left(s_{m}\right)}(x)=f_{m}^{\left(s_{m}\right)}(x), \quad \text { for } \quad m=1(1) n,
$$

where

$$
\chi_{m}^{\left(s_{m}\right)}(x)=\left\{\begin{array}{lr}
\sum_{i=1}^{n} \int_{0}^{x}\left(k_{m i}(x, t) \sum_{j=0}^{\beta_{m i}} y_{i}^{(j)}(t)\right) d t, & \text { if } s_{m}=0 \\
\sum_{i=1}^{n} \sum_{j=0}^{\beta_{m i}} \sum_{k=0}^{s_{m}-1} \mu_{k} \psi_{m i j}^{\left(s_{m}-k-1\right)}(x) y_{i}^{(j+k)}(x), & \text { if } \quad s_{m}=1,2, \ldots
\end{array}\right.
$$

$\mu_{k}, \eta_{k}$ are known constants and $\psi_{m i j}(x)=k_{m i}(x, x) q_{m i j}(x)$.

By substituting $x=0$, in (13), we have

$$
\sum_{i=1}^{n} \sum_{j=0}^{\alpha_{m i}} \sum_{k=0}^{s_{m}} \eta_{k} p_{m i j}^{\left(s_{m}-k\right)}(0) y_{i}^{(j+k)}(0)+\chi_{m}^{\left(s_{m}\right)}(0)=f_{m}^{\left(s_{m}\right)}(0), \quad \text { for } \quad m=1(1) n
$$

For $m=1(1) n, p_{m i j}^{\left(s_{m}-k\right)}(0)=0$, while $j+k>\alpha_{i}$, in $y_{i}^{(j+k)}(0)$, therefore the maximum derivative of order $y_{i}$, in (14) is $\alpha_{i}$.

In the first step of TSM, we suppose that the solution of (1) with the initial conditions (2) is giving by

$$
\mathbf{y}_{m}(x)=y_{m}(0)+y_{m}^{\prime}(0) x+\ldots+\frac{y_{m}^{\left(\alpha_{m}-1\right)}(0)}{\left(\alpha_{m}-1\right) !} x^{\alpha_{m}-1}+e_{m \alpha_{m}} x^{\alpha_{m}}, \quad \text { for } \quad m=1(1) n .
$$

By substituting (15) in (13) and setting $x=0$, we get

$$
\sum_{i=1}^{n} \sum_{j=0}^{\alpha_{m i}} \sum_{k=0}^{s_{m}} \lambda_{m i j k}+\psi_{m}^{\left(s_{m}\right)}(0)=f_{m}^{\left(s_{m}\right)}(0), \quad \text { for } m=1(1) n
$$


where

$$
\begin{aligned}
& \lambda_{m i j k}= \begin{cases}\left.\left(\alpha_{i}\right) ! e_{i \alpha_{i}} \eta_{k} p_{m i j}^{\left(s_{m}-k\right)}(0)\right), & \text { if } \alpha_{m i}=\alpha_{i}=j+k, \\
\eta_{k} p_{m i j}^{\left(s_{m}-k\right)}(0) y_{i}^{(j+k)}(0), & \text { otherwise, }\end{cases} \\
& \psi_{m}^{\left(s_{m}\right)}(0)= \begin{cases}0, & \text { if } s_{m}=0, \\
\sum_{i=1}^{n} \sum_{j=0}^{\beta_{m i}} \sum_{k=0}^{s_{m}-1} \gamma_{m i j k}, & \text { if } s_{m}=1,2, \ldots,\end{cases}
\end{aligned}
$$

and

$$
\gamma_{m i j k}= \begin{cases}\left(\alpha_{i}\right) ! e_{i \alpha_{i}} \mu_{k} \psi_{m i j}^{\left(s_{m}-k-1\right)}(0), & \text { if } \beta_{m i}=\alpha_{i}=j+k, \\ \mu_{k} \psi_{m i j}^{\left(s_{m}-k-1\right)}(0) y_{i}^{(j+k)}(0), & \text { otherwise. }\end{cases}
$$

Subtracting (14) from (16) and simplifying, we have the following system in matrix form

$$
A E=A Y,
$$

where $E=\left(\left(\alpha_{1}\right) ! e_{1 \alpha_{1}},\left(\alpha_{2}\right) ! e_{2 \alpha_{2}}, \ldots,\left(\alpha_{n}\right) ! e_{n \alpha_{n}},\right)^{t}, Y=\left(y_{1}^{\left(\alpha_{1}\right)}(0), y_{2}^{\left(\alpha_{2}\right)}(0), \ldots, y_{n}^{\left(\alpha_{n}\right)}(0)\right)^{t}$ and $A_{n \times n}$ is known matrix. Omitting linear dependent rows and columns $A_{n \times n}$ in (17), we have the following homogeny algebraic system

$$
B X=0
$$

where

$$
X=\left(\begin{array}{c}
y_{i_{1}}^{\left(\alpha_{i_{1}}\right)}(0)-\left(\alpha_{i_{1}}\right) ! e_{i_{1} \alpha_{i_{1}}} \\
\vdots \\
y_{i_{k}}^{\left(\alpha_{i_{k}}\right)}(0)-\left(\alpha_{i_{k}}\right) ! e_{i_{k} \alpha_{i_{k}}}
\end{array}\right)
$$

and $\left\{i_{i}, i_{2}, \ldots, i_{k}\right\} \subseteq\{1,2, \ldots, n\}$.

Therefore

$$
e_{i_{j} \alpha_{i_{j}}}=\frac{y_{i_{j}}^{\left(\alpha_{i_{j}}\right)}(0)}{\left(\alpha_{i_{j}}\right) !}, \quad j=1(1) k
$$

Now, we have to show that if (12) holds for $\sigma=\rho$, then it also holds for $\sigma=\rho+1$. Let the solution at $\sigma$ th step by TSM, be as follows

$$
\mathbf{y}_{m}(x)=y_{m}(0)+y_{m}^{\prime}(0) x+\ldots+\frac{y_{m}^{\left(\tau_{m}\right)}(0)}{\left(\tau_{m}\right) !} x^{\tau_{m}}, \quad \text { for } \quad m=1(1) n .
$$

Differentiating (13), $\left(\tau_{m}-\alpha_{m}\right)$ times, for $m=1(1) n$, then we have

$$
\sum_{i=1}^{n} \sum_{j=0}^{\alpha_{m i}} \sum_{k=0}^{\delta_{m}} \eta_{k} p_{m i j}^{\left(\delta_{m}-k\right)}(x) y_{i}^{(j+k)}(x)+\chi_{m}^{\left(\delta_{m}\right)}(x)=f_{m}^{\left(\delta_{m}\right)}(x), \quad \text { for } m=1(1) n,
$$

where $\delta_{m}=s_{m}+\tau_{m}-\alpha_{m}$ and

$$
\chi_{m}^{\left(\delta_{m}\right)}(x)=\sum_{i=1}^{n} \sum_{j=0}^{\beta_{m i}} \sum_{k=0}^{\delta_{m}-1} \mu_{k} \psi_{m i j}^{\left(\delta_{m}-k-1\right)}(x) y_{i}^{(j+k)}(x),
$$


$\psi_{m i j}(x)=k_{m i}(x, x) q_{m i j}(x)$ and $\mu_{k}, \eta_{k}$ are known constants.

In the same procedure that has been explained in (14)-(18), we can conclude that the equations (12) is also holds for $\sigma=\rho+1$.

\section{Modified Taylor-series method}

Let $\triangle=\left\{0=x_{0}, x_{1}, \ldots, x_{n}=b\right\}$, be an equidistance partition of $[0, b]$ where $s=x_{i+1}-x_{i}, i=$ $0,1, \ldots, n-1$ is the discretization parameter of the partition. In modified Taylor series method, we need to prove the following theorem..

Theorem 2: Let, the conditions of theorem (1) hold, and $k_{m l}(x, t)$ is a separable function, then there exist linear independent functions $\varphi_{0}(X), \ldots, \varphi_{\gamma}(X)$ and constants $c_{0}, \ldots, c_{\gamma}$ such that, $Y_{1}(X), Y_{2}(X), \ldots, Y_{n}(X)$ is the exact solution of following linear Volterra IDEs system

$$
\begin{gathered}
E_{m}(X+h)+V_{m}(X+h)+\sum_{k=0}^{\gamma} \delta_{m k} c_{k} \varphi_{k}(X)=f_{m}(X+h), \\
m=1(1) n
\end{gathered}
$$

with the initial conditions

$$
\begin{gathered}
Y_{k}(0)=y_{k}(h), Y_{k}^{\prime}(0)=y_{k}^{\prime}(h), \ldots, Y_{k}^{\left(\alpha_{k}-1\right)}(0)=y_{k}^{\left(\alpha_{k}-1\right)}(h), \\
k=1(1) n, \quad h \in \Gamma=[0, b]
\end{gathered}
$$

where

$$
\begin{aligned}
& E_{m}(X+h)=\sum_{l=1}^{n} \sum_{j=0}^{\alpha_{m l}} p_{m l j}(X+h) Y_{l}^{(j)}(X), \\
& V_{m}(X+h)=\sum_{l=1}^{n} \int_{0}^{X}\left(k_{m l}(X+h, u+h) \sum_{j=0}^{\beta_{m l}} Y_{l}^{(j)}(u)\right) d u,
\end{aligned}
$$

$Y_{l}(X)=y_{l}(X+h), l=1(1) n, X=x-h$ and $\delta_{m k}$, is equal 0,1 , or -1 .

Proof : $k_{m l}(x, t)$ is a separable function, therefore

$$
k_{m l}(x, t)=\sum_{k=0}^{\tau_{l}} v_{m l k}(x) w_{m l k}(t) .
$$

Substituting (25) into (1) and by change of variables $x=X+h$ and $t=u+h$, we have

$$
\begin{array}{r}
\sum_{l=1}^{n} \sum_{j=0}^{\alpha_{m l}} p_{m l j}(X+h) y_{l}^{(j)}(X+h)+\sum_{l=1}^{n} \int_{0}^{X}\left(k_{m l}(X+h, u+h) \sum_{j=0}^{\beta_{m l}} y_{l}^{(j)}(u+h)\right) d u \\
+\sum_{l=1}^{n} \sum_{k=0}^{\tau_{l}} v_{m l k}(X+h) a_{m l k}=f_{m}(X+h), \quad m=1(1) n,
\end{array}
$$

where

$$
a_{m l k}=\int_{-h}^{0}\left(w_{m l k}(u+h) \sum_{j=0}^{\beta_{m l}} y_{l}^{(j)}(u+h)\right) d u, \quad \text { for } \quad m, l=1(1) n, \quad k=0(1) \tau_{l} .
$$


Simplifying and classifying the last term on the left hand side of (26), we can write

$$
\sum_{l=1}^{n} \sum_{k=0}^{\tau} v_{m l k}(X+h) a_{m l k}=\sum_{k=0}^{\gamma} \delta_{m k} c_{k} \varphi_{k}(X), \quad \text { for } \quad m=1(1) n
$$

where, the known constant $\delta_{m k}$, is equal 0,1 , or $-1, \varphi_{0}(X), \ldots, \varphi_{\gamma}(X)$, are known linear independent functions, and also $c_{0}, \ldots, c_{\gamma}$, are unknown constants.

Therefore, by substituting $y_{l}(X+h)=Y_{l}(X)$, we can rewrite (26) in the form of (21).

We denote $\nu_{m}$ be the number of the unknown constants in (21), which are not appear in another equations for $m=1(1) n$. Now, for determining the unknown constants $c_{0}, \ldots, c_{\gamma}$, we need to take $\xi$ th derivative of relation (21) for $\xi=0, \ldots, \nu_{m}$, and then by setting $\mathrm{X}=0$, we will obtain the following algebraic system

$$
\left\{\begin{array}{l}
E_{1}(h)+V_{1}(h)+\sum_{k=0}^{\gamma} \delta_{m k} c_{k} \varphi_{k}(0)=f_{1}(h), \\
\vdots \\
E_{1}^{\left(\nu_{1}-1\right)}(h)+V_{1}^{\left(\nu_{1}-1\right)}(h)+\sum_{k=0}^{\gamma} \delta_{m k} c_{k} \varphi_{k}^{\left(\nu_{1}-1\right)}(0)=f_{1}^{\left(\nu_{1}-1\right)}(h), \\
\vdots \\
E_{n}(h)+V_{n}(h)+\sum_{k=0}^{\gamma} \delta_{m k} c_{k} \varphi_{k}(0)=f_{n}(h), \\
\vdots \\
E_{n}^{\left(\nu_{n}-1\right)}(h)+V_{n}^{\left(\nu_{n}-1\right)}(h)+V_{1}^{\left(\nu_{1}-1\right)}(h)+\sum_{k=0}^{\gamma} \delta_{m k} c_{k} \varphi_{k}^{\left(\nu_{n}-1\right)}(0)=f_{n}^{\left(\nu_{n}-1\right)}(h) .
\end{array}\right.
$$

In the case when the number of equations in (28) is less than $\gamma$, we need associate some more equations. these equation can be derived from those equations which are contain the more number of unknown constants, we consider by taking derivatives minimum times, solving the arising system of algebraic equations the constants $c_{0}, \ldots, c_{\gamma}$ can be determined. The existence of the solution of such system depend on the choosing of step length " $h "$.

For solving system of linear Volterra IDEs (1)-(2) by modified Taylor-series expansion method, let $h=$ $0, c_{i}=0, i=0,1, \ldots, \gamma$, by solving (21) and (22) by TSM, for $N$ iterations, we obtain

$$
y_{k}(x)=y_{k}(0)+\frac{y_{k}^{(1)}(0)}{1 !} X+\ldots+\frac{y_{k}^{\left(\alpha_{k}\right)}(0)}{\alpha_{k} !}, \quad k=1(1) n,
$$

where is Taylor expansion of the solution (1)-(2) at $h=0$.

At the next step, let $h=x_{1}$, from (28) and (29), we obtain $c_{i}, i=0,1, \ldots, \gamma$, and

$$
Y_{k}^{(j)}(0)=y_{k}^{(j)}\left(x_{1}\right), j=0,1, \ldots, \alpha_{k}+-1, \quad k=1,2, \ldots, n .
$$

By substituting the obtained values $c_{i}, \quad i=0,1, \ldots, \gamma$ into (21), we obtain a linear system of Volterra IDEs, by solving derived system, with TSM for $N$ iterations we have

$$
y_{k}(x)=y_{k}\left(x_{1}\right)+\frac{y_{k}^{(1)}\left(x_{1}\right)}{1 !} X+\ldots+\frac{y_{k}^{\left(\alpha_{k}+1\right)}\left(x_{1}\right)}{\left(\alpha_{k}+1\right) !}, \quad k=1(1) n
$$

where is Taylor expansion of the solution (1)-(2) at $h=x_{1}$.

By repeating the above step for $i=2,3, \ldots, n-1$, we can obtain Taylor expansion approximate (1)-(2) at $h=x_{i}$. 


\section{Estimation of error function}

We can easily check the accuracy of the solution obtained by present methods as follows. Let for $k=$ $1(1) n, e_{k}(x)=y_{k}(x)-\mathbf{y}_{k}(x)$, where $y_{k}(x), k=1(1) n$, is exact solution, and $\mathbf{y}_{k}(x), k=1(1) n$, is approximate solution (3).

Now, by substituting $y_{k}(x)=e_{k}(x)+\mathbf{y}_{k}(x)$, in (1)-(2), and rewriting we have

$$
\widehat{E}_{m}(x)+\widehat{V}_{m}(x)=\widehat{H}_{m}(x), \quad m=1(1) n ; \quad x, t \in \Gamma=[0, b],
$$

under the initial conditions

$$
e_{k}(0)=0, e_{k}^{\prime}(0)=0, \ldots, e_{k}^{\left(\alpha_{k}-1\right)}(0)=0, \quad k=1(1) n
$$

where

$$
\begin{aligned}
\widehat{E}_{m}(x) & =\sum_{k=1}^{n} \sum_{j=0}^{\alpha_{m i}} p_{m i j}(x) e_{k}^{(j)}(x) \\
\widehat{V}_{m}(x) & =\sum_{k=1}^{n} \int_{0}^{x}\left(k_{m i}(x, t) \sum_{j=0}^{\beta_{m i}} e_{k}^{(j)}(t)\right) d t
\end{aligned}
$$

and

$$
\widehat{H}_{m}(x)=f_{m}(x)-\sum_{k=1}^{n} \sum_{j=0}^{\alpha_{m k}} p_{m k j}(x) \mathbf{y}_{k}^{(j)}(x)-\sum_{k=1}^{n} \int_{0}^{x}\left(k_{m k}(x, t) \sum_{j=0}^{\beta_{m k}} \mathbf{y}_{k}^{(j)}(t) .\right.
$$

Now, by solving (32)-(33) with the manner which is given in the previous section, Taylor expansion approximate of the error function at $h=x_{i}, i=0, p, p+1, \ldots$ is obtained.

If $\epsilon=10^{-k}$ ( $\mathrm{k}$ is positive) is prescribed, then the truncation limit $N$ is increased until $e_{k}(x), k=1(1) n$, at each of the points $h=x_{i}, \quad i=0, p, p+1, \ldots$ becomes smaller than the prescribed $\epsilon=10^{-k}$.

\section{Convergence of the method}

From theorem 1. TSM and its modification expand the unknown functions $y_{i}(x)$ in (1)-(2) by its Taylor expansion as a polynomial degree $\mathrm{N}$

$$
\mathbf{y}_{m}(x)=\sum_{k=0}^{N} \frac{1}{k !} y_{m}^{(k)}(c)(x-c)^{k}+R_{m}(x, c), \quad m=1(1) n, \quad 0 \leq c \leq x,
$$

where the Lagrange remainder

$$
R_{m}(x, c)=\frac{y_{m}^{(N+1)}\left(\xi_{m}\right)}{(N+1) !}(x-c)^{N+1}
$$

for some $\xi_{m}$ between x,c. Moreover, suppose that there exist the maximum value $M_{m}$ of $y_{m}^{(N+1)}(x)$ on the interval $[0, b]$, then it can be proved that the Lagrange remainder has a bound independence of $\mathrm{x}$ and $\mathrm{c}$, i.e.

$$
\left|R_{m}(x, c)\right|=\frac{M_{m}}{(N+1) !}
$$

for two arbitrary points $\mathrm{x}$ and $\mathrm{c}$ on the interval $[0, b]$. Clearly, when $N$ is sufficiently large enough then, $R_{m}(x, c) \rightarrow 0$. 


\section{Applications and numerical results}

In this section, we show the efficiency of the modified TSM (MTSM) leads to higher accuracy by using some numerical results. We first use the MTSM in example 1 with detailed explanations and then in other example, we compare the purpose method with the other methods discussed in $[9,10]$. In Table 3, $e_{M T S M}, E e_{M T S M}, e_{D T M}$ and $e_{H V I M}$ stand for absolute error of MTSM, error estimation of MTSM, absolute error of differential transform method [10] and absolute error of He's variational iteration method [9] respectively.

Example 1. Consider a system of third-order linear Volterra IDEs on the interval $[0,6]$

$$
\left\{\begin{array}{l}
y_{1}^{\prime \prime}(x)+x^{2} y_{1}(x)-y_{2}^{\prime \prime}(x)+\int_{0}^{x}\left((x-t) y_{1}(t)+y_{2}(t)\right) d t=g_{1}(x), \\
4 x^{3} y_{1}^{\prime}(x)+6 x^{2} y_{1}(x)+y_{2}^{\prime \prime \prime}(x)+\int_{0}^{x}\left(y_{1}(t)+(x+t) y_{2}(t)\right) d t=g_{2}(x),
\end{array}\right.
$$

under the initial conditions, $y_{1}(0)=y_{1}^{\prime}(0)=1, y_{2}(0)=y_{2}^{\prime \prime}(0)=0, y_{2}^{\prime}(0)=1$,

where

$$
\begin{aligned}
& g_{1}(x)=\left(2+x^{2}\right) e^{x}-x-\cos (x)+\sin (x), \\
& g_{2}(x)=\sin (x)-(1+2 x) \cos (x)+e^{x}\left(1+6 x^{2}+4 x^{3}\right)+x-1 .
\end{aligned}
$$

The exact solution of this system is $y_{1}(x)=e^{x}, y_{2}(x)=\sin (x)$.

First of all, replace the functions $e^{x}, \sin (x), \cos (x)$, with theirs Taylor expansion in (37) at $x=0$. Corresponding TSM, we suppose that the solution of linear Volterra IDEs with the initial conditions be as follows

$$
\begin{aligned}
& \mathbf{y}_{1}(x)=1+x+e_{12} x^{2}, \\
& \mathbf{y}_{2}(x)=x+e_{23} x^{3},
\end{aligned}
$$

where $e_{12}$ and $e_{23}$ are unknown parameters.

Substitute (39) into (37), we have

$$
\begin{gathered}
-1+2 e_{12}+q_{1}(x)=0, \\
1+6 e_{23}+q_{2}(x)=0,
\end{gathered}
$$

where $q_{1}(x)$ and $q_{2}(x)$, are polynomials with degree greater equal than one.

By neglecting $q_{1}(x)$ and $q_{2}(x)$, in (40), we obtain $e_{12}=\frac{1}{2}$ and $e_{23}=-\frac{1}{6}$, therefore, the solution of (37) can be considered as

$$
\begin{aligned}
& \mathbf{y}_{1}(x)=1+x+\frac{x^{2}}{2}+e_{13} x^{3} \\
& \mathbf{y}_{2}(x)=x-\frac{x^{3}}{6}+e_{24} x^{4}
\end{aligned}
$$

where $e_{13}$ and $e_{24}$ are unknown parameters.

By substituting (41) in (37), we get

$$
\begin{aligned}
& \left(-1+6 e_{13}\right) x+q_{1}\left(x^{2}\right)=0, \\
& \left(24 e_{24}\right) x+q_{2}\left(x^{2}\right)=0,
\end{aligned}
$$

where $q_{1}\left(x^{2}\right)$ and $q_{2}\left(x^{2}\right)$, are polynomials with degree greater equal than two.

By ignoring $q_{1}\left(x^{2}\right)$ and $q_{2}\left(x^{2}\right)$ in (42), we obtain $e_{13}=\frac{1}{6}$ and $e_{24}=0$, therefore

$$
\begin{aligned}
& \mathbf{y}_{1}(x)=1+x+\frac{x^{2}}{2}+\frac{x^{3}}{6}, \\
& \mathbf{y}_{2}(x)=x-\frac{x^{3}}{6}
\end{aligned}
$$


By repeating the above procedure for $N=15$ iterations, we have

$$
\begin{aligned}
& \mathbf{y}_{1}(x)=1+x+\frac{x^{2}}{2}+\ldots+\frac{x^{16}}{20922789888000}, \\
& \mathbf{y}_{2}(x)=x-\frac{x^{3}}{6}+\frac{x^{5}}{120}-\ldots+\frac{x^{17}}{355687428096000} .
\end{aligned}
$$

That is 16 and 17, terms of Taylor expansion of exact solution $y_{1}(x)=e^{x}, y_{2}(x)=\sin (x)$, respectively at $x_{0}=0$.

Eq. (44), consider as approximate solution of (37) in the interval $[0,0.6]$.

By using theorem 2, we can covert linear Volterra IDEs system (37) to the following form

$$
\left\{\begin{aligned}
& Y_{1}^{\prime \prime}(X)+(X+h)^{2} Y_{1}(X)-Y_{2}^{\prime \prime}(X)+\int_{0}^{X}\left((X-u) Y_{1}(u)+Y_{2}(u)\right) d u \\
&+c_{0}(X+h)+c_{1}=G_{1}(X) \\
& 4(X+h)^{3} Y_{1}^{\prime}(X)+6(X+h)^{2} Y_{1}(X)+Y_{2}^{\prime \prime \prime}(X)+\int_{0}^{X}\left(Y_{1}(u)+(X+u+2 h) Y_{2}(u)\right) d u \\
&+c_{2}+c_{3}(X+h)=G_{2}(X) \\
&
\end{aligned}\right.
$$

where,

$$
\begin{gathered}
G_{1}(X)=\left(2+(X+h)^{2}\right) e^{X+h}-X-h-\cos (X+h)+\sin (X+h), \\
G_{2}(X)=\sin (X+h)-(1+2 X+2 h) \cos (X+h)+e^{X+h}\left(1+6(X+h)^{2}\right. \\
\left.+4(X+h)^{3}\right)+X+h-1,
\end{gathered}
$$

with the initial conditions

$$
Y_{1}(0)=y_{1}(h), \quad Y_{1}^{\prime}(0)=y_{1}^{\prime}(h), \quad Y_{2}(0)=y_{2}(h), \quad Y_{2}^{\prime}(0)=y_{2}^{\prime}(h), \quad Y_{2}^{\prime \prime}(0)=y_{2}^{\prime \prime}(h)
$$

and

$$
\left\{\begin{array}{c}
c_{0}=-1+e^{h}\left(2+2 h+h^{2}\right)+\cos (h)+\sin (h)-h\left(2 \mathbf{y}_{1}(h)+h \mathbf{y}_{1}^{\prime}(h)\right)- \\
\mathbf{y}_{1}^{\prime \prime \prime}(h)-\mathbf{y}_{2}(h)+\mathbf{y}_{2}^{\prime \prime \prime}(h), \\
c_{1}=e^{h}\left(2-2 h-h^{2}-h^{3}\right)-(1+h) \cos (h)+(1-h) \sin (h)+h^{2} \mathbf{y}_{1}(h)+ \\
h \mathbf{y}_{2}(h)+h^{3} \mathbf{y}_{1}^{\prime}(h)-\mathbf{y}_{1}^{\prime \prime}(h)+\mathbf{y}_{2}^{\prime \prime}(h)+h \mathbf{y}_{1}^{\prime \prime \prime}(h)-h \mathbf{y}_{2}^{\prime \prime \prime}(h), \\
c_{2}=-1+e^{h}\left(1-h-6 h^{2}-14 h^{3}-4 h^{4}\right)-(1+h) \cos (h)+ \\
\left(1-h-2 h^{2}\right) \sin (h)+h(1+6 h) \mathbf{y}_{1}(h)+2 h^{2} \mathbf{y}_{2}(h)+14 h^{3} \mathbf{y}_{1}^{\prime}(h)+ \\
4 h^{4} \mathbf{y}_{1}^{\prime \prime}(h)-\mathbf{y}_{2}^{\prime \prime \prime}(h)+h \mathbf{y}_{2}^{(4)}(h), \\
c_{3}=1+e^{h}\left(1+12 h+18 h^{2}+4 h^{3}\right)-\cos (h)+(1+2 h) \sin (h)-(1+12 h) \mathbf{y}_{1}(h)- \\
2 h \mathbf{y}_{2}(h)-18 h^{2} \mathbf{y}_{1}^{\prime}(h)-4 h^{3} \mathbf{y}_{1}^{\prime \prime}(h)-\mathbf{y}_{2}^{(4)}(h) .
\end{array}\right.
$$

Set $h=0.6$ into (45)-(47), and substitute the obtained values $c_{0}, c_{1}, c_{2}, c_{3}$, into (45). Then the arises linear system of Volterra linear Volterra IDEs with the initial conditions (46) can be solved by using our proposed methods for $N=15$, therefore we get the following approximate solution

$$
\mathbf{y}_{1}(x)=\sum_{i=0}^{17} e_{31 i}(x-0.6)^{i}, \quad \mathbf{y}_{2}(x)=\sum_{i=0}^{17} e_{32 i}(x-0.6)^{i},
$$

where $e_{31 i}, e_{32 i}, i=0,1,2, . ., 17$, are given in Table 1. Eq.(48) is an approximation for the Taylor expansion of (37) in neighborhood $x_{3}=0.6$, and consider as approximation solution of (37) in interval $(0.6,0.8]$.

Table 1.

The values of $e_{31 i}, e_{32 i}, i=0,1, \ldots, 17$. 


\begin{tabular}{cccccc}
\hline$i$ & $e_{31 i}$ & $e_{32 i}$ & $i$ & $e_{31 i}$ & $e_{32 i}$ \\
\hline 0 & $0.564642473395035 \mathrm{E}-0$ & $0.56464247339503 \mathrm{E}-0$ & 9 & $2.27440370068981 \mathrm{E}-06$ & $2.27440370068981 \mathrm{E}-06$ \\
1 & $0.825335614909678 \mathrm{E}-0$ & $0.82533561490967 \mathrm{E}-0$ & 10 & $-1.5560032889266 \mathrm{E}-07$ & $-1.5560032889266 \mathrm{E}-07$ \\
2 & $-0.28232123669751 \mathrm{E}-0$ & $-0.2823212366975 \mathrm{E}-0$ & 11 & $-2.0676397301677 \mathrm{E}-08$ & $-2.0676397301677 \mathrm{E}-08$ \\
3 & $-0.13755593581827 \mathrm{E}-0$ & $-0.1375559358182 \mathrm{E}-0$ & 12 & $1.17879036079657 \mathrm{E}-09$ & $1.17879036079657 \mathrm{E}-09$ \\
4 & $2.352676972479313 \mathrm{E}-2$ & $2.35267697247931 \mathrm{E}-2$ & 13 & $1.32541007939097 \mathrm{E}-10$ & $1.32541007939097 \mathrm{E}-10$ \\
5 & $6.877796790913993 \mathrm{E}-3$ & $6.87779679091399 \mathrm{E}-3$ & 14 & $-6.4768681440936 \mathrm{E}-12$ & $-6.4768681440936 \mathrm{E}-12$ \\
6 & $-7.84225657492964 \mathrm{E}-4$ & $-7.8422565749296 \mathrm{E}-4$ & 15 & $-6.3114612780652 \mathrm{E}-13$ & $-6.3114612780652 \mathrm{E}-13$ \\
7 & $-1.63757066450137 \mathrm{E}-4$ & $-1.6375706645013 \mathrm{E}-4$ & 16 & $2.69876049397373 \mathrm{E}-14$ & $2.69876049397373 \mathrm{E}-14$ \\
8 & $1.400402959817176 \mathrm{E}-5$ & $1.40040295981717 \mathrm{E}-5$ & 17 & $2.32048040828339 \mathrm{E}-15$ & $2.32048040828349 \mathrm{E}-15$ \\
\hline
\end{tabular}

By repeating the above procedure for $x_{i}=0.2 i, i=4,5, \ldots$, we can compute the approximation solution of (37) in the interval $(0.2 i, 0.2(i+1)]$. The numerical results in the Table 2. show the high accuracy of MTSM in the broad interval $[0,6]$, also Table 2. contains a numerical comparison between exact error of MTSM and estimation of error function that is introduced in section 4 .

Table 2.

Results for Example 1

\begin{tabular}{|c|c|c|c|c|}
\hline \multirow[b]{2}{*}{$x_{i}$} & \multicolumn{2}{|c|}{ Exact Error of MTSM } & \multicolumn{2}{|c|}{ Error Estimation of MTSM } \\
\hline & $e_{1}\left(x_{i}\right)$ & $e_{2}\left(x_{i}\right)$ & $E e_{1}\left(x_{i}\right)$ & $E e_{2}\left(x_{i}\right)$ \\
\hline 0.0 & 0 & 0 & 0 & 0 \\
\hline 0.5 & $4.44 \times 10^{-16}$ & 0 & $2.21 \times 10^{-20}$ & $2.06 \times 10^{-23}$ \\
\hline 1.0 & $8.88 \times 10^{-16}$ & $1.11 \times 10^{-16}$ & $1.42 \times 10^{-17}$ & $5.14 \times 10^{-18}$ \\
\hline 1.5 & $2.67 \times 10^{-15}$ & $1.11 \times 10^{-16}$ & $9.84 \times 10^{-17}$ & $3.66 \times 10^{-17}$ \\
\hline 2.0 & $5.33 \times 10^{-15}$ & $7.77 \times 10^{-16}$ & $3.57 \times 10^{-16}$ & $9.11 \times 10^{-17}$ \\
\hline 2.5 & $1.78 \times 10^{-15}$ & $1.77 \times 10^{-14}$ & $7.74 \times 10^{-16}$ & $1.16 \times 10^{-16}$ \\
\hline 3.0 & $3.55 \times 10^{-15}$ & $3.91 \times 10^{-14}$ & $1.23 \times 10^{-15}$ & $7.63 \times 10^{-17}$ \\
\hline 3.5 & $4.26 \times 10^{-14}$ & $2.47 \times 10^{-14}$ & $1.64 \times 10^{-15}$ & $2.29 \times 10^{-16}$ \\
\hline 4.0 & $6.40 \times 10^{-14}$ & $1.11 \times 10^{-13}$ & $2.01 \times 10^{-16}$ & $1.79 \times 10^{-15}$ \\
\hline 4.5 & $1.14 \times 10^{-13}$ & $7.84 \times 10^{-13}$ & $2.41 \times 10^{-14}$ & $2.73 \times 10^{-14}$ \\
\hline 5.0 & $2.56 \times 10^{-13}$ & $2.71 \times 10^{-12}$ & $8.52 \times 10^{-14}$ & $9.05 \times 10^{-14}$ \\
\hline 5.5 & $5.12 \times 10^{-13}$ & $6.78 \times 10^{-12}$ & $1.75 \times 10^{-13}$ & $1.89 \times 10^{-13}$ \\
\hline 6.0 & $1.71 \times 10^{-13}$ & $1.13 \times 10^{-11}$ & $3.36 \times 10^{-13}$ & $3.70 \times 10^{-13}$ \\
\hline
\end{tabular}

Example 2. Consider the following system of linear Volterra IDEs equations

$$
\left\{\begin{array}{r}
\sin (x) y_{1}^{\prime \prime}(x)+e^{x} y_{1}^{\prime}(x)+y_{2}^{\prime \prime}(x)+\int_{0}^{x}\left(y_{1}^{\prime}(t)+\cos (x-t) y_{2}(t)\right) \mathrm{d} t= \\
-2+e^{-x}+\left(-2+x+e^{-x}\right) \sin (x), \\
y_{1}^{\prime \prime}(x)+y_{1}^{\prime}(x)-x e^{x} y_{1}(x)-\cos (x) y_{2}^{\prime \prime}(x)+\int_{0}^{x}\left(e^{x+t} y_{1}^{\prime}(t)+x y_{2}(t)\right) \mathrm{d} t= \\
x\left(1-e^{x}\right)+2 \cos (x)(\sin (x)-x),
\end{array}\right.
$$

with the exact solution $y_{1}(x)=e^{-x}, y_{2}(x)=2 \sin (x)$ and $y_{1}(0)=1, y_{1}^{\prime}(0)=-1, y_{2}(0)=0, y_{2}^{\prime}(0)=2$, as the initial conditions.

By using TSM for $N=8$ iterations, the following approximate solution is obtained

$$
\begin{array}{r}
\mathbf{y}_{1}(x)=1-x+\frac{x^{2}}{2}+\ldots-\frac{x^{9}}{362880}, \\
\mathbf{y}_{2}(x)=2 x-\frac{x^{3}}{3}+\frac{x^{5}}{60}-\ldots+\frac{x^{9}}{181440} .
\end{array}
$$

That is 9 terms of Taylor expansion of the exact solution $y_{1}(x)=e^{-x}, y_{2}(x)=2 \sin (x)$, respectively at $x_{0}=0$. we consider (50) as the approximate solution (49) in the interval $[0,0.1]$.

By using theorem 2, we can convert (49) to the following linear IDEs system

$$
\left\{\begin{array}{c}
\sin (X+h) Y_{1}^{\prime \prime}(X)+e^{X+h} Y_{1}^{\prime}(X)+Y_{2}^{\prime \prime}(X)+\int_{0}^{X}\left(Y_{1}^{\prime}(u)+\cos (X-u) Y_{2}(u)\right) \mathrm{d} u= \\
-c_{0}-\cos (x+h) c_{1}-\sin (x+h) c_{2}-2+e^{-X-h}+\left(-2+X+h+e^{-X-h}\right) \sin (X+h), \\
Y_{1}^{\prime \prime}(X)+Y_{1}^{\prime}(X)-(X+h) e^{X+h} Y_{1}(X)-\cos (X+h) Y_{2}^{\prime \prime}(X)+\int_{0}^{X}\left(e^{X+u} Y_{1}^{\prime}(u)+(X+h) Y_{2}(u)\right) \mathrm{d} u= \\
-e^{X+h} c_{3}-(X+h) c_{4}+(X+h) e^{-x-h}+(X+h)\left(1-e^{X+h}\right)+2 \cos (X+h)(\sin (X+h)-X-h),
\end{array}\right.
$$


with $Y_{1}(0)=y_{1}(h), Y_{1}^{\prime}(0)=y_{1}^{\prime}(h), Y_{2}(0)=y_{2}(h), Y_{2}^{\prime}(0)=y_{2}^{\prime}(h)$, as initial conditions, and

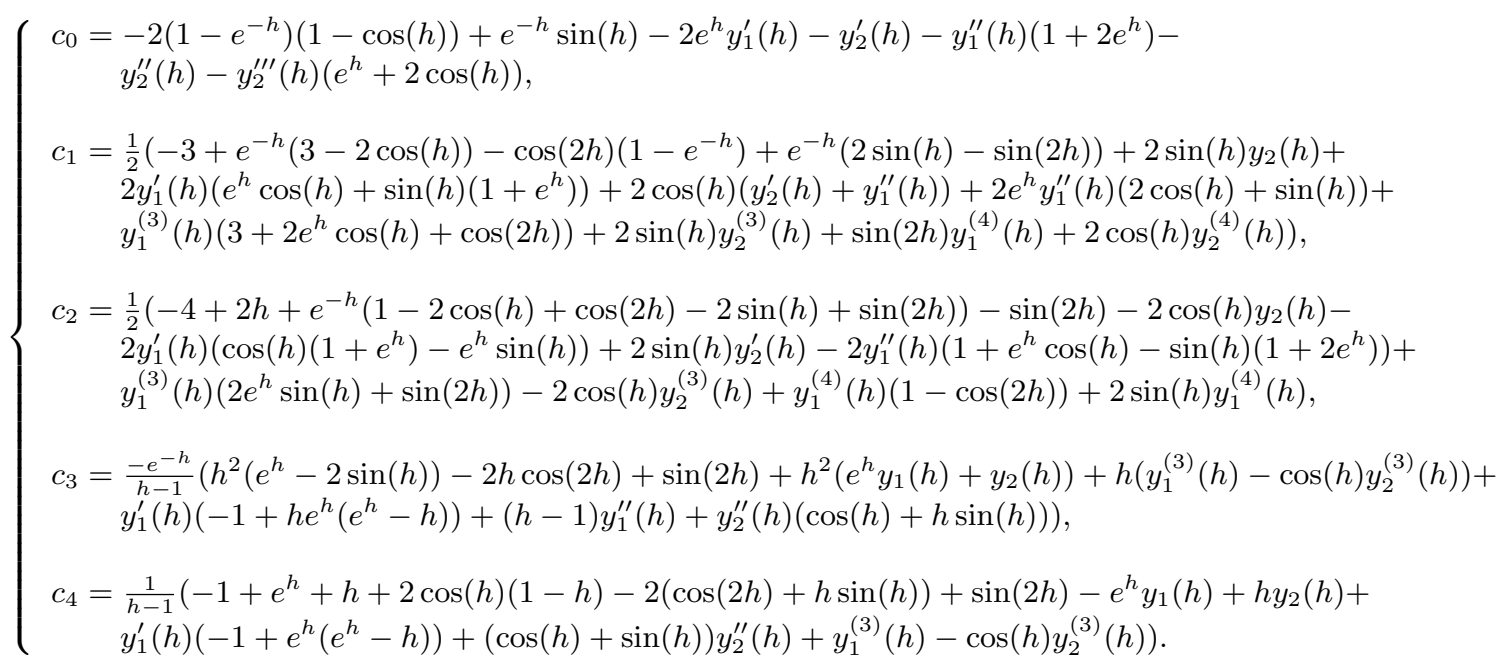

Now, by using the similar manner which is given in the Example 1. for $N=8$ iterations, we obtain an approximation of the exact solution and error function (49) in the intervals $[0.1 i, 0.1(i+1)], \mathrm{i}=1,2, \ldots, 9$. The numerical results in the Table 3. show that when the functions data are not polynomial, our approach are also applicable. 
Table 3.

Results for Example 2

\begin{tabular}{|c|c|c|c|c|}
\hline \multirow[b]{2}{*}{$x_{i}$} & \multicolumn{2}{|c|}{ Exact Error of MTSM } & \multicolumn{2}{|c|}{ Error Estimation of MTSM } \\
\hline & $e_{1}\left(x_{i}\right)$ & $e_{2}\left(x_{i}\right)$ & $E e_{1}\left(x_{i}\right)$ & $E e_{2}\left(x_{i}\right)$ \\
\hline 0.0 & 0 & 0 & 0 & 0 \\
\hline 0.1 & $1.11 \times 10^{-16}$ & $2.78 \times 10^{-17}$ & $2.75 \times 10^{-17}$ & $6.12 \times 10^{-21}$ \\
\hline 0.2 & $4.88 \times 10^{-15}$ & $2.22 \times 10^{-16}$ & $2.30 \times 10^{-15}$ & $7.47 \times 10^{-16}$ \\
\hline 0.3 & $3.91 \times 10^{-14}$ & $3.11 \times 10^{-15}$ & $1.60 \times 10^{-14}$ & $8.98 \times 10^{-15}$ \\
\hline 0.4 & $1.43 \times 10^{-13}$ & $9.66 \times 10^{-15}$ & $5.38 \times 10^{-14}$ & $3.51 \times 10^{-14}$ \\
\hline 0.5 & $3.58 \times 10^{-13}$ & $2.23 \times 10^{-15}$ & $1.25 \times 10^{-13}$ & $8.65 \times 10^{-14}$ \\
\hline 0.6 & $7.07 \times 10^{-13}$ & $7.86 \times 10^{-14}$ & $2.29 \times 10^{-13}$ & $1.60 \times 10^{-13}$ \\
\hline 0.7 & $1.17 \times 10^{-12}$ & $3.72 \times 10^{-13}$ & $3.46 \times 10^{-13}$ & $2.30 \times 10^{-13}$ \\
\hline 0.8 & $1.69 \times 10^{-12}$ & $1.14 \times 10^{-12}$ & $4.33 \times 10^{-13}$ & $2.42 \times 10^{-13}$ \\
\hline 0.9 & $2.10 \times 10^{-12}$ & $2.83 \times 10^{-12}$ & $4.14 \times 10^{-13}$ & $9.28 \times 10^{-13}$ \\
\hline 1.0 & $2.19 \times 10^{-12}$ & $6.15 \times 10^{-12}$ & $1.85 \times 10^{-13}$ & $3.81 \times 10^{-13}$ \\
\hline
\end{tabular}

Example 3. Consider the system of linear Volterra IDEs

$$
\left\{\begin{array}{l}
-y_{1}^{\prime}-\frac{1}{2} x y_{1}+\frac{3}{2} y_{2}=\frac{5}{2}-x-\frac{27}{2} x^{2}+x^{4}+\frac{3}{2}\left(-1+2 x^{2}\right)-\frac{1}{2} x\left(-3 x+4 x^{3}\right)+\int_{-1}^{x}\left(y_{1}-3 x y_{2}\right) \mathrm{d} t \\
\left.x^{2} y_{1}+y_{2}^{\prime}-x y_{2}=\frac{2}{5}+3 x+3 x^{3}-\frac{8 x^{5}}{5}+x^{2}\left(-3 x+4 x^{3}\right)+\int_{-1}^{x}\left((2 x+t) y_{1}+3 t^{2} y_{2}\right)\right) \mathrm{d} t
\end{array}\right.
$$

under the conditions $y_{1}(0)=0, y_{2}(0)=-1$, with the exact solution $y_{1}(x)=4 x^{3}-3 x, y_{2}(x)=2 x^{2}-1$. By using TSM for $N \geq 3$ iterations, the exact solution is obtained. In general, if the exact solution of linear Volterra IDEs system (1)-(2) is polynomial then TSM obtains the exact solution, for all $\mathrm{N}$ which is greater than degree of polynomial solution.

\section{References}

[1] T.L. Bo, L. Xie and X.J. Zheng, Numerical approach to wind ripple in desert, Int. J. Nonlinear Sci. Numer. Simul. 8(2) (2007) 223-228.

[2] F.Z. Sun, M. Gao, S.H. Lei, Y. B. Zhao, K. Wang, Y. T. Shi and N. H. Wang, The fractal dimension of the fractal model of drop-wise condensation and its experimental study, Int. J. Nonlinear Sci. Numer. Simul. 8 (2) (2007) 211-222.

[3] H. Wang, H.M. Fu, H.F. Zhang and Z. Q. Hu, A practical thermodynamic method to calculate the best glass-forming composition for bulk metallic glasses, Int. J. Nonlinear Sci. Numer. Simul. 8 (2) (2007) 171-178.

[4] L. Xu, J.H. He and Y. Liu, Electro spun nano-porous spheres with Chinese drug, Int. J. Nonlinear Sci. Numer. Simul. 8(2) (2007) 199-202.

[5] R. Agarwal and D. O'Regan, Integral and integro-differential equations theory, methods and applications, The Gordon and breach science publishers imprint, Singapore, Vol 2, 2000.

[6] K. Maleknejad, F. Mirzaee and S. Abbasbandy, Soling linear integro-differential equations system by using rationalized Haar functions method, Appl. Math. Comput. 155 (2004) 317-328.

[7] K. Maleknejad and M. Tavassoli Kajani, Solving linear integro-differential equation system by Galerkin methods with hybrid functions, Appl. Math. Comput. 159 (2004) 603-612.

[8] E. yusufoğlu, An efficient algorithm for solving integro-differential equations system, Appl. Math. Comput. 192 (2007) 51-55.

[9] J. Sabri-Nadjafi and M. Tamamgar, The Variational iteration method: A highly promising method for solving the system of integro-differential equations, Comput. and Math. with Applications 56 (2008) 346-351.

[10] A. Arikoglu and I. Oskol, Solution of integral and integro-differential equation systems by using differential transform method, Comput. and Math. with Applications 56 (2008) 2411-2417. 
[11] J. Biazar and H. Ghazevini, M. Eslami, He's homotopy perturbation method for systems of integro-differential equations, Chaos Solitons and Fractals 39 (2009) 1253-1258.

[12] E. yusufoğlu, Numerical solving initial value problem for Fredholm type linear integro-differential equation system, J. Franklin Inst. 346 (2009) 636-649.

[13] H. Aminikhah, A new analytical method for solving systems of linear integro-differential equations, J. of King Saud University(Science) doi:10.1016/j.jksus.2010.06.004.

[14] Y. Huang and X.-F. Li, Approximate solution of a class of linear integro-differential equations by Taylor expansion method, Int. J. Comput. Math. 87(6) (2010) 1277-1288.

[15] A. Karamete and M. Sezer, A Taylor Collocation method for the solution of linear integro-differential equations, Int. J. Comput. Math. 79(9) (2002) 987-1000.

[16] A. Tahmasbi and O.S. Fard, Numerical solution of linear Volterra integral equations system of the second kind, Appl. Math. Comput. 201 (2008) 547-552.

[17] L.M. Delves and J.L. Mohamad, Computational method for integral equations, Cambrige University Press, Cambrige, 1985

[18] Berenguer, M.I., Garralda-Guillem, A.I., Ruiz Galn ,M.: An approximation method for solving systems of Volterra integro-differential equations. Appl. Numer. Math. (2011, in press). doi:10.1016/j.apnum.2011.03.007 